\title{
XVII. \\ Beiträge zur Kenntniss maligner Tumoren an den äusseren Genitalien des Weibes.
}

\author{
(Aus dei Kgl. Universitäts-Frauenklinik zu Berlin. Direktor: Geh. Med.- \\ Path Prof. Dr. R. Olshausen.) \\ Von Dr. med. Richard Franke.
}

Herr Privatdocent Dr. Gebhard hat die Liebenswürdigkeit gehabt, mir das Material, welches das pathologische Institut der Frauenklinik an operirten Vulva- und Vagina-Carcinomen besitzt, zwecks einer mikroskopischen Untersuchung als Inaugural-Dissertation zur Verfügung zu stellen. Ich bearbeitete im Ganzen 20 Fälle, welche während der Zeit vom 16. Mai 1888 bis 30. Decbr. 1897 an der Klinik beobachtet wurden. Es sind dies nicht alle während des genannten Zeitraumes an der Anstalt behandelten derartigen Erkrankungen, da einerseits manche Patientinnen als inoperabel entlassen werden mussten, anderseits nicht von allen operirten Fällen noch Material in der pathologischen Sammlnng vorhanden war. Die verwahrten Tumoren waren theils in Formalin, theils in Alkohol conservirt und sämmtlich gut erbalten. Die excedirten Geschwülste aus neuester Zeit, welche von mir selbst mitbeobachtet worden sind, wurden von mir gleich nach der Operation in verschiedene Fixirungsflüssigkeiten gethan; es kamen zur Verwendung: Formalin, Alkohol in steigender Concentration, Zenker-Müller'sche Flüssigkeit, Flemming's Säuregemisch und Sublimat. Von allen Neoplasmen wurden sowohl Celloidin- als Paraffineinbettungen vorgenommen, die frischeren wurden zum Theil anf dem Gefriermikrotom geschnitten. Von Farbstofflösungen wurden angewendet hauptsächlich die Kernfarben Alaun-Carmin und Hämalaun ohne Contrastfärbung; daneben Hämatoxylin in Verbindung mit Eosin, Safranin zur Darstellung der Mitosen; van Gieson und Orcëin bei einem Hämangiosarcom. Es wurden von verschiedenen Stellen der Neubildungen Schnitte angefertigt, zum Theil wurden auch Serienschnitte hergestellt.

Unter den zwanzig von mir untersuchten Fällen befinden sich 13 Vulvaearcinome, 3 Vaginalcarcinome, 1 Urethralcarcinom, 1 polymorphzelliges Sarcom der Vulva, 1 Hämangiosarcom der Vagina und 1 Melanocarcinom der Vulva. Sämmtliche Tumoren sind primäre.

Es geht aus meiner Arbeit hervor, dass primär das Carcinom, speciell das Cancroid, die häufigste bösartige Neubildung an den äusseren weiblichen Zengungsorganen ist, während andere maligne Tumoren als eine grosse Seltenheit zu betrachten sind.

Was die drei letzten Fälle betrifft, so waren sie als gewöhnliche Carcinome makroskopisch diagnosticirt worden. Im Recidivstatus des MelanoArchiv f. pathol. Anat. Bd. 154. Hft, 2. 
carcinoms ist allerdings von einer, blauschwarzen" Prominenz dio Rede, ohne dass dieser verdächtigen Farbe eine besondere Bedentung zugelegt und der Tumor als melanotischer gedeutet worden wäre. Ebenso wurden die beiden Sarcome als solche nicht erkannt; erst bei meiner mikroskopischen Bearbeitung stellte sich der wahre Sachverhalt heraus.

In meiner Inaugural-Dissertation ${ }^{3}$ ) habe ich das Resultat meiner Untersuchungen veröffentlicht, wobei ich des Näheren auf die klinischen Erscheinungen und pathologisch-anatomischen Merkmale des Vulva- und Vagina-Carcinoms, dessen Aetiologie und Wesen eingegangen bin und die hauptsächlichsten Operationsverfahren erörtert habe. Am Ende der Arbeit - S. 98 bis 112 - befindet sich eine möglichst erschöpfende Zusammenstellung der Literatur über Vulva- und Vagina-Carcinome, umfassend die Jahre 1862 bis. $189 i^{\circ}$.

Hier beschränke ich mich darauf, nur 4 Fälle von malignen Tumoren, welche besonders interessant und hauptsächlich in Bezug auf ihre primäre Localisation als eine Seltenheit zu bezeichnen sind, aus meiner Arbeit mitzutheilen.

I. Fran Marie Rackow, 65 Jahre. Aufnahme 18. VIII. 85.

Anamnese: Frste Menses mit 18\% Jahren, regelmässig, stark, schmertos; Menopause seit 13 Jahren; 11 Geburten, Kind stets ausgetragen, letzte Geburt vor 29 Jahren. Patientin will jedesmal mit Zange entbunden worden sein; Wochenbett immer normal. Vor 22 Jahren in det Charité wegen inneren Leidens 6 Wochen behandelt. Vor 8 Tagen trat eine mässige Blutung aus den Genitalien auf. Patientin kommt wegen Drinbeschwerden nach der: Klinik.

Status praes: Die Harmöhrenmündung bildet einen breiten Spalt, der einem etwa taubeneigrossen, ulcerirten Tumor aufsitzt, welcher sich weiter anf die vordere Scheidenwand erstreckt. Genitalien klein; Oterus senil atrophisch, anteflektirt; Adnexe normal.

Operation am 21. VIII. Die Neubildung wird halbkreisförmig im Gesunden umschnitten, sodam unter geringer Blatung von der Symphyse stumpf abgetrennt. In der Scheide werden die lateralen Schnitte durch eine quere Incision vereinigt und der Tumor so frei gemacht, dass er nur noch an dex Wurzel der Harnöhhre hängt. Diese wird dicht an der Blase durchschnitten. Hieranf wird durch mehrere tiefgreifende Catgutnähte die noch übrige Urethralschleimhaut mit dem äusseren Wundrande vereinigt, welcher dadurch stak nach innen retrahirt wird. Die unteren Wundränder werden mit zwei Nähten seitlich geschlossen, sodass von der nea gebildeten Harnöbre aus seitlieh qwei Wundränder nach aussen riehen.

3. IX. Ringe um die Haxmöbre eine Zone von gut granulirendem Gewebe, das nach aussen und innen in Narbenzïge übergeht, Keine Beschwerden ausser incontinentia uriaae, welche vom Tage der Operation an besteht.

1) Mikroskopische Untersuchungen über maligne Tumoren der Vulva und Vagina mit besonderer Berücksichtigung des Cancinoms. Berlin. Mai 1898. S. 112. 
5. IX. Patientin wird mit der Weisung entlassen, sich poliklinisch wieder einmal vorzustellen.

Mikrosk op. Befund. Das Epidermisepithel ist in Grossen und Ganzen gut erhalten. Wenn es auch an einigen Stellen eine geringe Zerklüftung und Desquamation zeigt, so steigert sich dieselbe doch nirgends bis zar Freilegung des Bindegewebes. Es sendet die bekannten Epithelstränge in die Tiefe des Stromas, welches reichlich von Epithelzügen dnrchwuchert wird, ohne seinerseits eine merkliche Proliferation erkennen zu lassen. Die polymorphen Carcinomzellen vercinigen sich in dem weitaus grössten Theile des Präparates zu einer typischen aveolären Anordnung mit zum Theil recht breiten, zum Theil recht schmalen Bindegewebssepten. Oefters wird der aveoläre Bau von einem mehr tubulären abgelöst, und in der ganzen Ausdehnung des Bildes finden sich, wenn auch nicht in grösserer Zahl, Epithelzapfen und Perlen in den verschiedensten Stadien einer regressiven Metamorphose. Einige von ihnen zeigen einen centralen Zerfall, und ihr Centrum weist, zwecks Resorption der Detritusmassen, eine reichliche Leukocytenansammlung auf, die theilweise auch bereits wieder in Auflösung begriffen ist. Sonst ist die Leukocyteninfiltration von der gewöhnlichen Intensität, nur eine geringe Abweichung vom allgemeinen Schema zeigt sich darin, dass die Rundzellen in der Gegend der stärksten Ausbreitung des Tumors einen ausgesprochenen Zerfall darbieten, worin die Theorie eine Stütze findet, dass die weissen Blutkörperchen, welche gewissermassen als schützender Damm gegen die Ueberschwemmung des Gewebes durch die andrängenden Carcinomzẹllen aufgeworfen werden, von den wachsenden Carcinomzellen zerstört und zur Nałrung aufgebraucht würden, sodass also eine Phagocytose im entgegengesetzten Sinne stattfindet, d. h. nicht wie gewöhnlich die Lymphzellen, sondern die anlockenden, gleichsam als Fremdkörper fungirenden Neoplasmazellen die Rolle von Phagocyten spielen. Plattenepithelcarcinom²).

II. Frau Emma Trews, 62 Jahre. Aufnahme 16. VIII. 97.

Anamnese: Menopause seit 16 Jahren. Im Oktober vorigen Jahres entwickelte sich an der r. grossen Labie eine Geschwulst, die im Januar dieses Jahres in Bromberg entfernt wurde. Seit Pfingsten besteht wieder eine Geschwulst an beiden grossen Labien und Anschwellung der Inguinaldrüsen rechts und links, welche auf Salbenbehandlung zeitweise zurückgegangen sein soll.

Status praes.: Gut entwickelte Fran von leidlichem Kräfte- und Ernährungszustand. Die untere Hälfte der 1. grossen Labie ist in einen kleinapfelgrossen, ziemlich circumscripten Tumor von harter Konsistenz und etwas höckeriger Oberfläche verwandelt. Die der anderen Labie zu-

2) Was die Frequenz, Genese und Operation der primären und secundären carcinomatösen Erkrankungen der, Vulva und Vagina so benachbarten, Urethra anbelangt, so rerweise ich auf meine Dissertation S. 40 u. 41. 
gekehrte Fläche des Tumors ist in der Ausdehnung eines $Z_{w}$ eimarkstückes ulcerirt. Das Ulcus ist grangrünlich, schmierig belegt und leicht blutend. An der entsprechenden Stelle der $r$. grossen Labie ein über haselnussgrosser Knoten von der gleichen Konsistenz. Die Haut über demselben ist nicht ulcerirt, lässt sich aber ebensowenig wie links in Falten aufheben. Faginaluntersuchung wird nicht vorgenommen. Die Inguinaldrüsen sind beiderseits geschwollen und zu grossen Packeten vereinigt, nicht druckempfindlich.

19. VIII. Opuration: Nach oberflächlicher Kauterisation mittelst Raquelin wird zunächst der 1. Tumor im gesunden Gewebe exstirpirt. Derselbe zeigt sich mit der vorderen Rectumschleimhaut verwachsen und muss mit dem Messer unter Kontrole eines in den Mastdarm eingeführten Fingers abgetrennt werden. Die Darmwand selbst bleibt intakt. Oberflächliche Verschorîng der Wundfläche mit dem Thermokauter. Sodann Exstixpation des Tumor labii majoris dextri, wobei mehrere spritzende Arterien umstochen werden müssen. Durch versenlite and oherfluchliche vähte werden beide Defecte geschlossen. Nummehr folgt die Ausräumung der Inguinaldrüsen. Dieselben erweisen sich als sehr morsch, im Innern zerfallen; einzelne reissen ein und entleeren weissen, breiigen Inhalt. Mebrere grössere Venen, die mit den Drüsempacketen fest zusammenhängen, werden nach vorheriger Abbindung durehtrennt. Es besteht num beiderseitig eine ziemlich tiefe Wundhöhle mit theilweise unterminirter Haxt. Die Hautränder werden dnrch wenig tiefgraifende Enopfnähte aneinander gebracht, und dann die Haut durch komprimirenden Verband lest in die Wundtaschen hineingedrückt. 0,01 Morph. hydrochl. subcutan injizirt.

Entlassungsbefund 17. IX. 97: Im Bereich der I. Inguinalwande mebrere leicht ulcerirende, mit etwas Eiter belegte Partien; desgleichen am unteren Rande der Scheidenwande eine alcerirte Stelle; geringer Ausfluss. Patientin wird als geheilt entlassen.

Mikroskop. Befund. Das Oberhantepithel ist überall gut erhalten und seine Grenze gegen das Bindegewebe ohne pathologische Veränderungen; nirgends sendet es Epithelstränge in die Tiefe. Die dusbreitung des Neuplasmas ist eine vollkommen diffuse und reicht bis dicht an das Epidermisepithel heran, ohne an einer einzigen Stelle in dasselbe überzugehen; es bricht in Gegentheil die Wucherung der veoplastischen Zellen meist plötzlich $a b$, eine neutrale Zone zwischen sich und dem Deckepithel freilassend. Die Geschwulst gehört zur Gruppe der Bindesubstanzneoplasmen, des Sarcoms, dessen Zellen einerseits bezüglich ikrer Zahl, anderseits bezüglich ihrer Dimensionen der Zwischensubstanz gegenüber vollkommen vorherrschend sind, sodass ein Bild entsteht, welches den unentwickelten Bindesubstanzen, dem embryonalen Keingewebe, ähnelt. Der vorliegende Tumor erweist sich als äusserst zellreich und ganz bosonders arm an Intercellularsubstanz, trägt also einen medullären Charalter. Die Gefässe ziehen als dünnwandige Kanäle zwischen den Zellmassen hindurch, 
ohne hyperplastisch oder ektatisch zu sein und ohne dass ihre Wandzellen in die Zellen des Neoplasma continuirlich übergingen. Dasselbe setzt sich aus den verschiedensten Zellen, sowohl hinsichtlich ihrer Gestalt als ihrer Ausdehnung znsammen. Am meisten vertreten findet man zwei Arten von.Zellen, welche sich wesentlich durch ihre ganz verschiedene Grösse von einander abheben. Die einen sind klein und enthalten einen kugeligen, bisweilen etwas ovalen, verhältnissmässig sehr grossen Kern, welcher von einer winzigen Menge Protoplasma umgeben wird. Die anderen sind von weit bedeutenderem tmfang und schliessen in einer sehr erheblichen Masse von Protoplasma einen oder zwei, mitunter anch mehrere, sehr grosse runde Kerne ein, wobei sie mitunter einen epithelialen Charakter tragen. Reichlicher sieht man sodann noch Spindelzellen, welche meist recht klein und protoplasmaarm sind, so dass die aus ihren Polen austretenden Fasern scheimbar aus den Kernen kommen (Kernfasern). Nur ausnahmsweise findet man etwas voluminösere Spindelzellen. Die kleinen sind oft zu Bündeln gruppirt, indem sie sich mit ihren Breitseiten aneinander gelegt haben. Diese Fascikel sind bald längs, bald schräg, bald quer von dem Schnitte getroffen, ein Zeichen, dass sie sich nach verschiedenen Richtungen durchflechten. Bisweilen sind sie um die Gefässe herum angeordnet, sodass sie letztere wie Lymphscheiden umgeben. Hicr und da zeigen sich zwischen den beschriebenen Zellformationen zerstreut auch wohl noch sternfö́rmige Gebilde, ohne ihre verschiedenen Fortsätze, die sie nach allen Richtungen aussenden, zu einem Netzwerk zu vereinigen, wie es bei den Lymphosarcomen der Fall ist. Sodann erkennt man wieder ganz unregelmässig geformte Zellen, welche eine Gestalt angenommen haben, wie sie durch den ihnen zur Verfügung stehenden Raum bedingt wird. Riesenzellen finden sich nirgends im Präparat. Die Infiltration des Gewebes mit Rundzellen ist eine mässige, und sie sowohl als die Sarcomzellen zeigen auf der einen Seite bereits einen z. Th. beginnenden, z. Th. fortgeschrittenen Zerfall, auf der anderen Seite erblickt man zahlreiche typische und vereinzelte atypische Mitosen der Neoplasmazellen. Der beschriebene Tumor dürfte also wohl zu den Medullarsarcomen mit polymorphen Zellen zu rechnen sein.

Mikroskop. Befund der Lymphdrüsen: Die Lymphdrüsen, welche in ihrem Centrum einen starken käsigen und fettigen Zerfall mit Haemorrhagien zeigen, sind vollständig sarcomatös entartet. Man kann in ihnen dieselben verschiedenen Zellvariationen, auch in dem nämlichen Verhältniss wie im Primärtamor, unterscheiden mit der einzigen Ausnahme, dass vielleicht die mehrkernigen, grossen Sarcomzellen im Vergleich mit der Zusammensetzung des primären Neoplasmas etwas prädominiren. Die Tumorzellen durchsetzen die Lymphdrüsen diffus, z. Th. innerhalb der Lymphspalten, zu kürzeren und längeren Strängen aneinander geschlossen, sich fortschiebend und die lymphatisehen Elemente verdrängend. Die Umgebung der Lymphdrüsen ist noch nicht afficirt. 
III. Frau Emilie Eggert, 55 Jahre. Aufnahme 30. IV. 1889, zum zweiten Mal am 30. VII. 1889.

Anamuese: Patientin ist seit dem 18. Lebensjahr menstruirt; die Menses waren regelmässig, schwach, zweitägig, mit Schmerzen im Unterleib verbunden. Die letzte Regel vor 6 Jahren. 4 normale "Geburten. 1873 Pocken; sonst nie emsilich krank. Mitte Februar plötzlich eine starke Blutung ans den Geschlechtstheilen, seit Aufang März Ausfluss und Schmerzen in Unterleib, Rücken und Brust, besonders heftig aber im Rectrum. Seit 1866 Hämorrhoiden.

Status praesons: Introitus und Scheido mittelweit, letztere kurz, senil veründert. Uterus retrovertirt, von Seiten der Adnexa nichts Pathologisches nachweisbar. Dicht hinter dem Introitus befindet sich in der Mitte der hinteren Scheidewand ein etwa markstückgrosses Geschwür mit anfgeworfenen Rändern, speckigem Grunde und harter Consistenz. Die harte Infiltration setzt sich nach beiden Seiten der Vagina, besonders aber nach links fort, sodass das gesammte infiltrirte Gebiet etwa Thalergrösse erreicht. Die Inguinaldrüsen sind nicht geschwollen. Per rectum fühlt man, dass der carcinomatöse Knoten unmittelbar unter der Rectm-Sehleimhaut gelegen ist, doch ist letztere noch über dein Tumor yerschieblich. Am Anus zahlreiche Varicen.

3. V. Operation durch Olshausen: Das Carcinom wird an seinom oberen Raude von der gesunden Vaginalwand abgetrennt, seitlich umsclinitten und ron oben herab lospräparirt; dabei Blutung aus einer grösseren Anzahl von Venen und einigen Arterien, die abgeklemmt, beziehentlich unterbunden werden. Vom Rectum ans wird die Loslösung unit den Finger controlirt. Die Trennung geht in lockerem Gewebe vor sich, greift von dex Mitte des Uleus aus direkt auf die Darmwand äber, deren Muscularis freigelegt wird. Eintretende Defaecation macht mehrfach Carbolirrigation erforderlich. Umschneidung des unteren Carcinomrandes. Das Perineum wird in seiner vorderen Hälfte durch einen in der Raphe verlaufenden Sehnitt durchtrennt. Bei der Auslösung des Carcinomgrandes entsteht eine fast markstückgrosse Oeffntung in der Rectalwand, dieselbe wird durch 7 Catgutligaturen geschlossen. Eine fortlaufende Catgutnaht am oberen Mundwinkel beginnend, $2 \mathrm{~b}$ nnd wieder aufsteigend und nochmals nach unten gefïhrt, vereinigt den Defect. Bei der Operation grosser Blutverlust und eintretende beträchtliche Anämie.

7. V. Patientin klagt über Drängen gegen den After und über diffuse Leibschmerzen. Abdomen etwas aufgetriebon. Lebhafte Berregung der mit Gas angefüllten Darmsehlingen durch den Leib hindurch zu sthen und zu fühlen. Ricintzöl. Mehrfach Strhlentleerungen.

14. V. Patientin hatte in den letzten Tagen wiederholentlich Stuhl; hente Nuchmittag tritt Koth durch die Vagina aus. Die Untersuchung ergiebt, dass das Rectum seht harte Faecesballen enthält. Dieselben werden unter. Wasserspülnng mit dem Finger sorgfältig entfernt. Es scheint 2 Finger breit über dem Anus eine für einen Finger curehgängige 
Communication zwisehen Darm and Scheide zu bestehen, doch wird von einer genaueren Untersuchung Abstand genommen. 3 mal täglich $21 / 2$ proc. Carbolspülung der Vagina.

21. V. Seit 2 Tagen gehen keine Faeces mehr per Vaginam ab; eine Fistel ist nicht zu entdecken.

27. V. Entlassungsbefund: Zwischen Vagina und Rectum ein bis $1 \mathrm{~cm}$. dickes, weiches Septum. Vaginalfläche in einer Ausbreitung von etwa $2 \mathrm{qcm}$. granulirend. Analverschluss gut functionirend. Patientin hat beständig das Gefühl, als ob sie einen Ring trage. Keine besonderen Schmerzen. Entlassung.

30. VII. Patientin kommt heute wieder in die Klinik, weil dieselben Symptome, wie vor der Operation (Schmerzen im Unterleib, in der Lendengegend und im Rectum, sie belästigen. Stuhlgang regelmässig, ohne Beschwerden.

Status praes.: Von der Operation ist in der Vagina nur die narbige Einziehung ihrer Rückenwand zu sehen. Ausserdem findet sich etwa $4 \mathrm{~cm}$ oberhalb des Introitus linkerseits eine halberbsengrosse Granulation, anscheinend von einem daselbst befindlichen Seidenfaden herrührend; derselbe wird mit der Pincette entfernt. Die Rectalmucosa ist ein wenig eingezogen. $1 \mathrm{~cm}$ hoch im Rectum findet sich auf der linken Seite anscheinend in der Submucosa ein halbhaselnussgrosser Knoten, von dem einige zarte, verschiebliche Stränge, höchstwahrscheinlich Lymphgefässe, nach oben und vorn verlaufen. In der Narbe selbst liegt ein kleiner, erbsengrosser, harter Knoten, unweit von jenem grösseren.

2. VIII. Operation durch Olshausen: Exstirpation der Carcinomknoten. Der Anus wird links vorn incidirt und stumpf ein Weg zur haselnussgrossen Metastase gebohrt. Auslösung derselben unter Vordrängen von der Vagina aus. Entfernung mehrerer kleinerer Knoten aus dem Septum rectovaginale. Vernähung der Wundhöble durch fortlaufende Catgutnähte.

4. VIII. Die Gegend zwischen hinterer Hälfte der 1. grossen Labie, Oberschenkel, Perineum und Anus ist halbhandtellergross infiltrirt und blau verfärbt.

8. VIII. Wunde am Anus ist auseinandergewichen und hat $\mathrm{z}$. Th. blutige, z. Th. jauchige Massen austreten lassen. Vorsichtige Ausspülung. Aufpulverung von Jodoform.

30. VIII. Wunde granulirt gut. Ausspülung.

3. XI. Wunde gut granulirend und retrahirt. Faeces können nicht gehalten werden. Entlassung.

Mikroskop. Befund: Das Epidermisepithel ist überall vollkommen intakt, seine Grenze gegen das Corium nirgends verwischt. Das Bindegewebsstroma wird spärlich von längeren und kürzeren Strängen mit polymorphen, theils platten, theils kubischen, theils cylindrischen Zellen durchzogen. Diese Stränge weisen meistens ein Lumen auf, theilweise erinnern sie auch an solide Drüsenkolben und Beeren und verleihen dem 
Tumor einen den Adenomen nnd gewissen Carcinomen äbnlicheu Bau. Wenn nnn auch das Präparat allerdings reichlich wirkliche Drüsenschläuche aufweist, so kann es sich doch weder um ein gutartiges Adenom handeln, da u. A. zu einem solchen die Zahl der Drüsenwucherungen viel zu gering wäre, noch um ein Adenocarcinom, weil nirgends eine Entartung der cylindrischen, specifischen Drüsenbestandtheile nachweisbar ist. Ein Careinom von alveolärer oder tubulärer Structur, das vom Deckepithel oder den Wurzelscheiden der Haarbälge ausgegangen wäre, ist schon deshalb ansgeschlossen, weil, abgesehen vom Fehlen jeglicher Epithelzapten und Perlen und abgesehen von der Vielgestaltigkeit der Zellen, nirgends ein Zusammenhang der Tumormassen mit dem im Gegentheil nicht im Geringsten afficirten Plattenepithel nachweisbar ist. Fehlen $s 0$ für eine Epithelgeschwulst jegliche Anbaltspunkte, so sind sie zu der Annahme eines Bindesubstanz-Neoplasma desto zahlreicher und beweisender vorhanden. Es dürfte sich hier um ein Sarcom mit organoidem Bau, speciell um ein Alveolär- oder Tubulärsarcom handeln, Geschwülste, in denen die zelligen, zuweilen auffallend epithelännlichen Bestandiheile, welche das Charakteristicum des Tumors ausmachen, in bestimmten Gruppen zusammenliegen. Wie in den meisten Fällen der sareomatösen Neoplasmen mit besonderer Anordnung und Gruppirung ihrer specifischen Bestandtheile, so hängt auch in dem vorliegenden Falle der tubuläre Bau in ausgesprochener Weise mit der besonderen Genese des Neoplasmas zusammen. Es kann nehmlich bei derartigen Neubildungen der Beweis erbracht werden, dass sie vorwiegend bald von den Endothelien der Lymphgefässe, bald von denen der Blutgefässe ausgehen, sodass man einerseits ein Lymphangiosarcoma ${ }^{3}$ ), anderseits ein Haemangiosarcoma unterscheiden kann. Diese evidente Abstammung hat bekauntlich zur Aufstellung einer besonderen Geschwulstform, des Endothelioms, geführt, weiches wieder, je nachdem man die Endothelien entsprechend ihrer embryonalen Herkunft den Epithel- oder Bindesubstangewebe zuzählt, von den einen Autoren den Carcinomen, von den anderen den Sarcomen zugezählt wird. Das vorliegende Präparat zeigt also diesen tubulären Bå von drüsenartigen Kanälen und soliden Zelìsträngen. Man sieht die verschiedensten Stufen und Variationen der Endothel-Entartung der Blutgefässe, z. Th. beginnt eben die Wucherung, z. Th. sind die Lumina bereits compact mit Zellen vollgepfropft, wobei theilweise auch, in Folge Cebergreifens der whohemden Zeilen auf das unmittelbar benachbarte Bindegewebe, eine Verbreiterung des Gefässquersehnittes zu constatiren ist. Nirgends ist, zu eruiren, dass die Wucherung von den Zellen der Tunica adventitia ausginge, wie sie Waldeyer ${ }^{4}$ ) für sein "Angiosarcom" beschreibt, ein Ausdruek, welchen dieser Anatom zuerst anwandte und für Geschwülste vorschlug, deren Characteristicum "in der Entwickelung von den Gefäss-

3) Kolaczek: Veber das Angiosarcom. Deutsche Zeitschr. f. Chirurgie. IX und XIII.

4) Die Entwickelung der Carcinome. Dieses Archiv. Bd. 55. 
adventitien aus mit der Beibehaltung des gefässähnlichen Verlaufes der Zellstränge" gegeben würde. Diese Sträuge sind in der Mehrzahl im Querschnitt getroffen, hinter denen die Zahl der Längsschnitte zurïcksteht. Manche Gebilde sind auf's Stricteste beweisend, dass die maligne Neubildung von den Endothelien der Gefässe ausgeht, und zwar solehen kleinen Kalibers, d. h. kurz vor ihrem Uebergang in Capillaren. So zeigt das Präparat z. B. des öfteren folgenden Befund: Man sieht ein in den Längssehnitt gefallenes Gefäss, dessen Intima aus langgestreckten, spindelförmigen, längsverlaufenden Endothelzellen und einer structurlosen Haut, der sogenaunten elastischen Innenhaut, bestelat, daran schliesst sich die Tunica media mit vereinzelten glatten Ringmuskelfasern, auf welche nach aussen die Adventitia folgt, bestehend ans feinfaserigen, längsgerichteten Bindegewebszügen und feinsten elastischen Fascrn und ohne scharfe Grenze in das die Blutbahn umscheidende Bindegewebsstroma übergehend. Der Bau des Gefässes ist also bis hierher ein ganz normaler zu nennen. Dann aber beginnt, mitunter ziemlich plötzlich, das Endothel zu wuchern sowohl nach dem Iumen zu, dasselbe allmählich verstopfend, als auch die Media und Adventitia durchsetzend, sndass die Figur einer Keule entsteht, deren Griff das noch normal gebaute Gefäss, deren Kopf der Theil des entarteten Gefässes mit dem wuchernden Endothel darstellt. Inwieweit sich auch eine Entartung der Lymphgefässendothelien an dem Aufbau des Neoplasmas hetheiligt, lasse ich dahingestellt. Leukocyten liegen nur vereinzelt im Gesichtsfelde. Es dürfte sich also in dem vorliegenden Falle wohl um ein Angiosarcom, bezw. Endotheliom handeln.

IV. Frau Augusta Klein, 68 Jahre. Aufnahme 30. IX. 97 u. 6. XII. 97.

Anamnese: Seit dem 13. Lebensjahr ist Patientin immer regelmässig, aber spärlich menstruirt gewesen. Seit 18 Jahren Menopause. Patientin hat 3 Kinder geboren, wovon das orste mittelst Zange entwickelt werden musste. Luetzte Geburt vor 27 Jahren; kein Abort. Kurz vor Beginn der Regel hat Patientin stets starke Kopfschmerzen mit Erbrechen gehabt, das sich mit dem Eintritt ins Klimakterium verlor. Patientin leidet seit 3/4 Jahren an blutigem Ausfluss, der bald schwächer, bald stärker wird. Seit 6-8 Wochen hat der Fluor stetig an Intensität zugenommen und es haben sich Schmerzen im Unterleib dazu gesellt. Urinabsonderung, Defäcation, Appetit, Verdauung, Sehlaf gut. Wegen der erwähnten Schmerzen und des Zunehmens des Ausflusses sucht Patientin die Klinik auf.

Status präsens: Im Allgemeinen recht guter Ernährungszustand; stark entwickelter panniculus adiposus. Am Lab. maj. dextrum findet sich ein klein-handtellergrosser, flacher, harter, an der Peripherie höckeriger, auf der breiten Oberfläche kraterförmig vertiefter, ulcerirter Tumor, der nach oben bis zum Ansatz der Clitoris geht, unten noch $2 \mathrm{~cm}$ vom Introitus vaginae frei lässt. Die hinter dem Tumor liegende Vaginalschleimhaut weist einzelne lnollige Verdiekungen auf; auch auf dem linken Beckenboden sind Verdickungen zu fühlen. Linke Labien frei. Rechte Ingninaldrüsen wallnussgross zu fühlen, die linken sind nicht zu palpiren. 
2. X. Operation durch Koblanek: Verschorfung des Carcinoms besonders des Randes, mittelst des Paquelins; Circumcision, die oben bis hart an die Urethra reicht. Ausschneiden des Caroinoms unter Compression des verletzten Schwellkörpers der Clitoris. Die nicht erhebliche Blutung wird durch vereinzelte Unterbindungen gestillt. Fortaufende Catgutnaht. Collodium. Schnitt parallel zum Lig. Pouparti dextrum; das subcutane Fett wird mit den darin eingeschlossenen Drüsen als ungem fähr faustgrosses Packet ausgeschät. Mit zwei fortlaufenden Catgutnähten wird das untere Zellgewebe vereinigt. Durch die Cutis ebenfalls fortlautende Catgutnaht, Collodium, Gaze, Wickelverband. Dauer der Operation eine Stunde, 0,01 Morph. hydr. subcut.

16. X. Ausschälung eines apfelgrossen Atheroms aus der linken behaarten Kopfseite, 0,01 Morph, bydrochl. subcutan injicirt.

30. X. Wunde an der Vulva ist gut verheilt. Narbe sehx derb, in der Nähe der Clitoris leichtere Verhärtung. Ebenso zeigt die sonst gut verheilte Wunde der Regio inguinalis dextra an einzelnen Stellen derbe Infiltrationen des Gewebes. Wunde am Kopf ist per primam intentionem geheilt. Patientin wird als geheilt entlassen.

Patientin komml am 6. XII. 97 wegen Bückfalls des alten Leidens Fieder in die Anstalt.

Status präsens: Am Harmö̈hrwulst eine, plauschwarze", etwa kirschkerngrosse Prominenz, die von intacter Schleimhaut überzogen und ron fest-weicher Consistenz ist, Der Tumor wird bei dex verdächtigen Anamnese als Carcinomrecidiv angesprochen. In der r. Inguinalgegend unter der Narbe eine unbestimmte Härte zu palpircn.

9. XII. Operation durch Koblanet: Die nohw färote Geschwulst wird umschnitten nnd abpräparirt, der Defekt durch fortanfende Catgutnaht geschlossen. Spaltung der Haut der Regio inguinalis dextra; aus dem Unterhautbindegewebe wird ganz nabe der grossen Tene eine etwas härtere Partie excidirt. Naht der Hautwade, Verband.

21. XII. Die Wunde an der Urethra ist nicht geheilt, sondern ulcerint in der Tiefe; rechts von derselben eine hochrothe, indurirte, bohnengrosse Parfie, die als entründliche Inflitration angesprochen wird. Die Wunde in der linken Leistenbenge ist per primam geheilt. Patientin wird octlassen und anf den 4. 1. 98 zur Besichtigung wieder bestelt.

Als Patientin sich wieder vorstellte, wurden nur Granulome der Narben diagnosticirt, so dass sie nicht in die Klinik zu einer Operation aufgenorimen wurde.

Rei einer spateren Vorstellung in der Poliblinilk hatte das Recidiy bereits soweit in der Richtung des Beckeninnern um sich gegriffen, dass eine Operation als aussichtslos bezeichnet werden musste. Seit einigen Wochen hat Patientin sich wegen fortsehreitenden Marasmus in ein Berliner städtisches Krankenhaus aufnehmen lassen, in welchem mit Sicherheit eine Metastase in der Lunge diagnosticirt werden lonnte. An den Unterextremitäten nat sich allmählich, höchst wahrobeinlich in folge 
Ausbreitung der Neubildung im kleinen Becken, ein so hochgradiger Hydrops anasarka entwickclt, dass Skarifikationen vorgenommen werden mussten, um die Beschwerden einigermaassen erträglich zu gestalten. Das Recidiv an der Vulva ist nur klein-kirsehengross. ${ }^{\circ}$ )

Mikroskop. Befund: Das geschichtete Epidermisepithel ist zwar stets an einer bestimmten Stelle in den zahlreichen Sehnitten, die durch den Rand der bei der ersten Operation entfernten Geschwulst gelegt wrixden, als solches noch sicher za crkennen, doch auch an dieser ist es theilweise schon recht erheblich desquamirt usd verändert. Dieser noch erhaltene Pest zeigt eine Strecke weit seine normale Grenze gegen das Bindegewebe, allmählich aber fangen die untersten Lagen der Schleinschicht an, atypiseb zu wuchern, und es schieben sich, immer mehr an Ausdehnung zunehmend, viele theils schmale, lange, theils breite, kurze Epithelstränge unter leblafter, mit reichlicher Gefäss-Hyperplasie verbundener Bindegewebs-Wucherung unregelmässig zwischen die Balken des Stromas. Die Ausdehnung dieser Cancroidstränge bleibt im Grossen und Ganzen eine ziemlich oberflächliche, indem dieselben bald in mässiger Tiefe, ohne irgendwelche Modifikationen einzugehen, theis stumpf, theils zugespitzt enden. Schon an dieser circumscripten Stelle fallen strotzend mit Blut gefüllte Capillaren und kleinere Gefässe (Formalinhärtung) und neben den Leukocyten $z$ war nieht im grossen Haufen vereinigte, aber überall hier und da im Gewebe zerstrente, ausserhalb der Gefässbahn gelegene rothe Blutbörperchen ins Auge. Diese Hyperämie erreicht dann ziemlich plötzlich eine bedeutende Höhe und steigert sich zu grösseren Blutextravasaten. In gleichem Maasse rimmt die Rundzellen-Infiltration zu, welche unter ausgesprochenem Zerfall einen so bohen Grad erreicht, dass von den Zellen der hier aufs unregelmässigste zerklüfteten und zerrissenen, entarteten Epidermis fast nichts mehr zu sehen ist und ein den diphtherischen Endzündungen ähnliches Bild entsteht. Die ganze Oberfläche des Präparates manifestirt sich so fast ausschliesslich als eine ziemlich breite Auflagerung von Detritusmassen, welche der Hauptsache nach ans zerfallenen weissen und rothen Blutkörperchen und Fibrinfäden bestehen und in ihren tieferen Lagen von erweiterten und hyperplasirten Capillaren und kleineren Blutgefässen durchzogen werden, eine Erscheinung, welche zum Theil auf die ulcerirte Oberfläche, zum Theil auf die Ustion des Tumors mit dem Paquelin, welche vor der Operation zur Vermeidung einer Infection der Wunde mit Krebskeimen vorgenommen wurde, zurückzuführen ist. Mit zunehmender Entfernung von der Oberfläche werden die Entzündungserscheinungen all-

5) Patientin ist Anfang Juni gestorben. Die Section hat sehr viele ausgedehnte Metastasen von meist tiefschwarzer Farbe in den verschiedensten Organen ergeben. Nochmals angestellte mikraskopische Untersuchungen der metastatischen Knoten haben eine Bestätigung der Diagnose ergeben, welche ich an dem durch die erste Operation entfernten Tumor gestellt habe. Präparate davon sind in einer Sitzung der medicinischen Geselischaft zu Rerlin gezeigt worden. 
mählich geringer, obwolll das ganze Präparat in mässiger Intensität durchziehend, und man erkennt unter der immer noch sehr reichlichen Infiltration von Leukocyten allmählich deutlicher ein engmaschiges Netz von wuchernden Epithelzellen, welches in seinen untersten Auslänfern verhältnissmässig wenig von Rundzellen durchsetzt ist, sondern rein als alveoläres Uarcinom mit sehr dünnen, wenig Kerne enthaltenden Bindegewebssepten zu erkennen ist. Jo mehr das Carcinom in die Tiefe greift, desto zarter und dünner werden die einzelnen Netzfäden, so dass sie sich schliesslich der Mehrzahl nach aus länge an einander gereihten Zellen nur noch zusammensetzen. Das Neoplasma erreicht im Vergleich zu seiner sonst so ausgesprochenen Proliferations-Intensität eine nur mässige Tiefe, indem es nicht bis an dic Knäueldrüsen heranwuehert, sondern bereits vorher meist plötglich abbricht, ohne, einige wenige Stellen ausgenommen, auch nur minimale Reaction seitens des noch nicht carcinomatös entarteten Gewebes hervorzurufen. Nirgends zeigt die Neubildung in den angefertigten Schnitten Verhormung oder hyaline Eratartung seiner polymorphen, grossen, lebhaft gefärbte Kerne einschliessenden Zellen. Es sind überhaupt weder Zapfen noch Perlen zwischen den alveolären Bau eingestreut zu finden, sondern die Wucherung hat streng seinen typischen Charaliter als Alveolärcarcinom bewahrt, welches sich unter Anderem hauptächlich durch das Fasergerüst, welches die Zellen einschliesst, von Sarcom noterseheidet,

Zwiselen den farblosen Geschrulstrellen findet man nun reichlich solche, welche Pigmonthörner in ihrem Innern beherbergen. Disse Zellen zeigen häufig in einer gelblich bis gelbbraun gefärbten Grundmasse zahlreiche, ziemlich grosse, schwarze Grasula in der Mehrzahl von annähernd gleichen Yolumen. Der Kern der Zelle terhält sich dabei cehr versehieden. In den einen Zellen besteht er fast durchweg aus dorselben tiefschwarzen Nasse, wie die im Zellprotoplasma eingesehlossenen Pigmentkömer. Fr hat dabei seine runde, bläschenformige Beschaffenheit alls Epithelkexn vollständig bewahrt. Bei starker Vergrösserming - Oelinmersion $1 / 1 n$, Oeular I - zeigt er eine geringe Aederung, indem weniger gefurbte, etwas gesehlängelte Linien, z. Th. annähernd parallel verlaufend, z. Th. sich zt einem Netzwerk verschlingend, durch die diffuse dunkelschwarze Materie hindurchriehen. Andere Z mit schwarzen Pigmentikörnehen und lassen, aller Wahrscheinlichkeit nach in Folge der Menge des Pigmentes, kein als Thern deutbares Gebilde mehr unterscheiden. Eine Entfärbung mit Chromsäure zur Sichtbarmachung des Kernes wurde nicht vorgenomtnen. Wieder andere Zellen zeigen einen gana normalen, soliden, etwas ovalen Epithelkern mit migheren Kerns.öperchen und um denselben herum Pigmentkügelchen gruppirty $z_{*}$ Th. das Protoplasma überall gleiohmässig durchsetzend, z. Th. eine halbmondförmige Figur darstellend, bisweilen die Peripherie des Keñes oder des Protoplasmas sauroartig umziehend, so dass man den Eindract erhäit, als ob eine von Pigmentgranula durchsetzte Membran die zienlich sehtyach gefärbte Innensubstanz rmgäbe. Sodaxn sieht man auch folgende Bilder: 
In der Mitte der Zelle, mitunter dem Rande derselben etwas genähert, erkennt man einen ganz blass gefärbten, rundlichen Fleck (Kern?) und um denselben herum den Zelleib dicht mit Pigmentkörnchen ansgefülit, so dass eine den Ehrlich'shen Mastzellen ähnliche Figur entsteht. Ferner zeigen die Geschwulstzellen die verschiedensten Debergänge der Pigmentirung von völliger Pigmentlosigkeit bis zum stärksten Pigmentgehalt: die einen Zellen haben nur einen blassgelblichen bis hellbraunen Ton ohne Granula, Befunde, wolche zweierlei Auslegungen zulassen. Entweder der Farbstoff hat zunäuchst den Zelleib gleichmässig durchtränkt, da derartige Zellen nicht selten bei der Berliner Blan-Reaction eine diffuse blangrünliche Färbung zeigen, so dass man annehmeñ muss, dass der eisenhaltige Farbstoff sich zuerst in gleichmässiger Ausbreitung innerhalb der Zelle befindet und wahrscheinlich erst später in Form von Körnchen ausgeschieden wird; oder es hat die andere Annahme Geltung, nach welcher die diffuse helle Färbung als ein Product secundärer Veränderung des Farbstoffes aufzufassen ist, indem die Zelle in Zerfall und das Pigment in Auflösung begriffen ist. Andere Zellen zeigen eben die Bildung von Granula, mitunter nur ein einziges anfweisend; noch andere zeigen bereits eine grössere Anhärufung von Pigmentkügelchen; wieder andere sind so vollgepfropft mit ihnen, dass sie sich bei schwacher Vergrösserung als diffuse schwarze Klümpchen documentiren. Mitunter zeigt auch nur der Kern Veränderungen von einem gelblichen Schimmer bis zu einer tiefschwarzen Fürbung. Unter diesen den Uebergang bildenden Zellen findet man ab und zu neben bereits völlig schwarzen Pigmentkörperchen solche von der nämlichen Grösse und Form, welche noch pigmentlos die zur Verwendung gekommenen Farbflüssigkeiten, wenn auch etwas schwächer, angenommen haben, wozu ich gleich bemerken will, dass eine Verwechselung mit polynukleolären Leukocyten nicht vorliegt. ${ }^{6}$ ) Pigmentkörner von Form und Durchmesser eines halben, ganzen und doppelten rothen Blutkörperchens, Befunde, wie sie manche Autoren gemacht und zur Stütze der hämatogenen Theorie verwendet haben - direkte Aufnahme ganzer und zusammengeflossener rother BlutLörperchen oder grösserer Trümmer derselben - habe ich nirgends sehen können. Alle diese Pigmentzellen zeigen sich zwischen den einzelnen Geschwulstelementen zerstreut, meist mehr nach der Tiefe zu, doch findet man sie hier und da auch in die obersten Strata des wuchernden Epithels verfolgbar. Dass die farbstofíhaltigen Zellen eigentliche Geschwulstzellen sind, ergiebt sich am deutlichsten ans folgendem Befunde: In der Tiefe des Tumors, und zwar in der Nähe seines Randes gegen das Stroma hin, da wo die Epithelstränge, nur noch ans einzelnen kettenartig längs aneinandergereihten Zellen bestehend, sich vorwärtsschieben, sieht man verschiedentlich ein solches Glied einer Kette auch von Pigmentzellen dargestellt, so dass also wohl kaum noch ein Zweifel aufkommen kann, ob es sich hier wirklich um eigentliche Gesehwulstzellen handle. Diese pigmenthaltigen Zellen sind ziemlich unregelmässig vertheilt,

5) Eine Deutung dieser Erscheinung unterlasse ich. 
eine besondere Gruppirung, eța eine perivasculäre oder um die vorhandenen Eaemorrhagien herum, wie man es häufig bei Melayosarcomen findet, lässt sich nirgends erkennen; im Gegentheil ist die Pigmentirung der ZeIlen in der Gegend der blutreichen Gefässe and Extravasate eine auffailend geringe, meist sind diese Bezirke pigmentfrei. Es wechseln überhaupt grössere pigmenthaltige Stellen mit pigmentlosen im Präparat sehr untereinander ab; manche Schnitte sind fast ohne alles Pigment. Im Grossen und Ganzen jedoch liegen die farbigen Zellen gleichmässig in der Geschwalst zerstrent, nur selten za klcineren Convoluten zusammengeballt. Oefters sind die Pigmentzellen, speciell die am intensiysten gefärbten, etwas grösser als die farblosen. Dabei haben sie zum weitans grössten Theil inhe epitheliale Form bewahrt, nur ganz vereinzelt sind sie von etwas unregelmässiger Gestalt, ma man kann dann dreieckige, längliche, sichelformige, scheibenförmige und elliptische Figuren unterscheiden. Hier und da findet man auch in Zerfall begriffene Pigmentzellen. Eine Pigmentirugg der Bindegewebselemente habe ieh nirgends nachweisen können, im Gegentheil schliessen die farbigen Geschwulstzellen mit dem Rande der epithelialen Wucherung. schar? $a b$, ohne auf das unterliegende Gewebsstroma mit seinen ovalen bís spindelförmigen Zellen tiberzugreifen. Ebeuso wenig finden sich unter den spärlichen Zellen der schmalen Bindegewebssepten, welche die alveolenäbnlichen Züge der Geschwulstmasse trennen, irgend to pigmenthaltige.

In meiner Dissertation ${ }^{7}$ ) bin ich nach Schilderung der morphologischen Ferhaltnisse der Pigmentoellen des $\mathbb{N}$ aheren anf den jetzigen Stand ${ }^{8}$ der Frage nach der chemischen $Z \mathrm{Znsmmensetzung}$ und der Abstammang des Farbstoffes eingegangen and habe die Resultate dor bis jetzi erschienenen Arbeiten einer kritischen Besprechng untexsogen.

In meinem Fali von Melanocarcinom habe ich durch Ferro- und Ferricyankalium in Verbindung mit Salzsärare auf Eisen gefahndet, doch war es mir nie möglich, die Berliner Blaureaction zu erhalten, während sie an anderen von mir untersuchten Organen, welche Blnteisen vermuthen liessen, stets, bei frühzeitigex Yoraahme der Untersuchung, gute, charalkteristisehe Bilder gegeben hat, sodass ich also den Yorwurf und das Bedenken, welche von mancher Seite gegen diese Methode erhoben wotden sind, als nicht stichhaltig zurückweisen kann. Etwas anderes ist die Frage, ob hämosiderinhaltige Pigmentkörner mit der Zeit in solche übergehen tönnen, welche keine Eisenreaction mehr geben. Ich glaube allerdings in Uebereinstimmung mit $\mathrm{Neumann}{ }^{9}$ ) and Sehmidt ${ }^{10}$ ), dass die Eisen-

7) $5.77-87$.

s) Hinsichtlich ausführlicher Iiteraturangaben vermeise ick auf die letzte knitische Zusammenstellnng derselben, welche v, Bueklinghans en i. d. dentsch. Chir. Lief. 2 und 8, S. 443 Pr., gegeben hat.

9) Beitrăge zur Kenntniss d. patholog. Pigmente. Dies. Arch., Bd.111, 1888.

16) Ueber die Verwandtschaft der hämatogenen und antochthonen Pigmente und deren Stellnng z. Hämosiderin. Dieses Archiv, Bd. 115. 
reaction der Hämosiderine nicht von Bestand ist, da nicht selten unter Verhältnissen, unter denen man die Hämosiderinreaction erwarten darf, dièselbe negativ ist, sodass man nicht umhin kann anzunehmen, dass in eisenhaltigen Pigmentkörnern sich allmählich Veränderungen vollziehen, wobei das Eisen entweder aus dem Pigment abgeschieden wird oder eine Verbindung eingeht, die den mikrochemischen Nachweis sehr erschwert oder überhaupt unmöglich macht. Anzunehmen, dass die Eisenreaction der Hämosiderine von Anfang an fehlen kann, scheint mir mehr als zweifelhaft; wohl aber kann sie bei der einen oder anderen Methode missglücken, je nachdem das Eisen im Hämosiderin gebunden ist Die erste Möglichkeit, dass das Pigment bereits in eine mikrochemisch nicht mehr nachweisbare Eorm übergegangen wäre, kann für meinen Fall nicht statthaben, da die Probe kurze Zeit nach der Operation angestellt wurde. Wohl aber ist der Umstand in Rechnung zu ziehen, dass das Eisen sich vielleicht in einer Verbindung befand, welche mit Blutlaugensalz und Salzsäure dasselbe nicht erkennen lässt. Eine Reaction mit einem anderen chemischen Stoffe wurde nicht angestellt, da ich nach den in meiner Dissertation angesteliten Erörterungen dem Eisennachweise in melanotischen Pigmenten keinen besonderen Werth beilegen zu müssen glaube. In Folge des negativen Ausfalles meiner Eisenreaction konnte ich mein Augenmerk nicht auf eine Beobachtung verschiedener Autoren richten, welche darin bestand, dass bei positiven Eisenreactionen mitunter mehr Kerne sichtbar zu werden scheinen, als rorher zu sehen waren, eine Wahrnehmung, welche sie in dem Sinne auslegten, dass neben pigmenthaltigen Eisenkörnern noch farblose Eisen-Albuminate in den Zellen liegen müssen. Auch der vorliegende Tumor enthält, wie in den meisten derartigen Fällen, Zerfallsprodukte rother Blntkörperchen, welche ohne Ausnahme die Reaction mit Ferrocyankalium gaben. Ein nicht zu unterschätzender Factor zur Stätze meiner Behauptung, dass es sich in dem vorliegenden Falle um ein Melanocarcinom handelt, d. h. die Pigmentzellen eigentliche Geschwulstzellen sind, ist neben dem milkroskopischen Befunde der, dass das durch die zweite Operation entfernte Recidiv auch makroskopisch dentlich das Aussehen der melanotischen Tumoren zeigte, was bei der nach Veröffentlichung meiner Dissertation stattgefundenen Obduction noch deutlicher hervorgetreten ist. Es lässt sich nehmlich oft die Wahrnehmung machen, dass der primäre Tumor weniger pigmentirt ist, sodass er sich makroskopisch nicht als melanotischer kennzeichnet, während die Metastasen eine braune bis schwarze Earbe zeigen.

Die bei der ersten Operation exstirpirten Lymphdrüsenpackete zeigen sich ebenfalls melano-carcinomatös entartet. Die Epithelzellen wuchern z. Th. um die Lymphdrüsen in kleinalveolärer Anordnung herum, z Th. entwickeln dic Neoplasmamassen sich auch innerhalb der Lymphbahnen aus eingeschleppten Krebszellen, indem sie in Haufen und Strängen zusammengelagert sind, welche die Lymphocyten verdrängen. Diese Krebszellnester werden theilweise von einem aus dem Stützgewebe der Lymphdrüsen hervorgegangenem Bindegewebsstroma umschlossen. Auch hier 
finden sich grössere Blutextravasate, and welchseln pigmentfreie und pigmenthaltige Carcinomzellen mit einander ab. Letatere sind in relativ bedeutend reichlicher Menge als im Primärtumor vorhanden.

ob eine pigmentirte Warze oder sonst irgend eine kleine Pigmentanomalle durch atypische Wucherung seiner Zellen den Ausgangspunkt für dis maligne Geschwalst abgegeben hat, lässt sich nicht mehr eruiren. In der Krankengeschichte finden sich keine diesberäglichen Angaber.

Es ist djes die erste Beobachtung eines primären Melanocarcinome an der. Vulva und der 16. Fall yon melanotischen Tumoren an den äusseren weiblichen Genitalien einschliesslich Schamhügel nnd Perineum; denn bis jetat existiren nur die folgenden 15 derartigen Veröffentlichuogen:

1. Precott-Hewettin). Melanotische Geschmulst an den Schamlippen einer 59jabrigen Frau. Sie hat sich ans einem an dieser Stelle seit mehreren Jahren bestehenden Fleck in seln raschem Wachsthum entwickelt; Verjanchung, Blutung, heftige Schmerzen, Operation, nach 6 Monaten Exitus. IT. KIob ${ }^{19}$ ): Melanotische Camoinom-Tumoren an den Labien einer alten, an melanotischer Carcinotnatose voratorbenen Frau. III. Bailly ${ }^{13}$ ): 72 jährige Fran, bei welcher sich ein Melanom der $x$. $k$. Labie zeigte. Dasselbe hatte sich innerhalb 10 Monaten an Taubeneigrösse ontwickelt und rerstopfte das Orificum vulvae vollständig. Palliativoperation

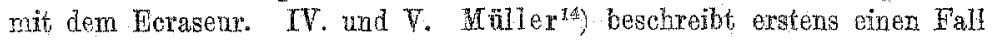
mit einem gänseeigrossen melanotischen Sareom an der Citoris. Exstirpation mit Ansnahme der infilturten Inguinaldrïscn, welche später entterat werden sollten. Die Frau ging rasch zu Grunde in Folge ausgedehnter Netastasen, unter Anderem auch in Douglas-Raum und im Lig. rotundum, wie die dutopise ergab. Zweitens theilt en einen Fall von melanotischem Sarom bei einem 39jahrigen Fräkleis mit; an dex Imnendlüche der kleinen Sabie, otwa $1 \mathrm{~cm}$ ron der Clitoris entfernt, fand sich eine prall gesparinte, wallnussgrosse, bläulich durchschimmerade Gaschwulst, wrelohe sich gegen die Ungebung deutlich abgrenzen liess. Die Leistendrusen zeigten sich intakt, Operation; recidivfrei noch nach 2 Jahren. VT. Fischeris beobachtete ein melanotisches, nlcerirendes Sarcom bei einer 56 jahrigen Frau an dem linken grossen Labinm. Dasselbe wuchs viermal bis Walnassgrösse, platzte damn und entleerte etwas Eiter und Blut. Firstirpation; nach wenigen Wochen Recidir; Tumonen in der Benchhohle: Kranle wird

1) Melanosis of the labium and glans, of the groine and pubes. Lancet. March. 1861. Ref. in Canstatt's Jahresbericht f. 1861. Bd. IV. 1. 261.

32) Patil. Anat. d. weibl. Sexualorgane. 1864, p. 467.

13) Gazette hebdomadaire. 2 Sér. V. (IV). No. 7. 1868. Ref. ron Diekrich in Langenbeck's Arch. Bd. 36. p. 295 .

14) Zur: Casuistik d. Neubild, an d. ausa. weibl. Genit. Berliner klin. Woehenschr. 1881. S. 446 u. S. 448.

15) Veber die Ursachen der Krebskrankheit und ihre Heilung durch das Messer. Deutsche Zeitschr, f. Chir. Nd.14. 8. 248. 
als unheilbar entlassen; bald laxitus. VII. Goth ${ }^{16}$ ): Fall von Melano sarcoma alveolare, mannsfaustgrosse Geschwulst, stellenweise oberflächlich ulcerizt und aus zwei Theilen bestehend, localisirt auf beiden kleinen Labien, lem Praeputium, dem hinteren Rand des Introitus und der hinteren Vaginalfläche. Keine Drüsen-Infiltration, Exstirpation, nach 5 Wochen kein Rückfall. VIII. Terrillo ${ }^{17}$ ). Nussgrosses Sarcom an der Innenfläche des rechten Lab. mipns. Die Schleimhaut der Umgebung des Tumors, ebenso die der Vagina und Vaginalportion zeigt dunkle Pigmentirung. Abtragung mittelst Thermokanters. $\$ / 4$ Jahr post operationem Exitus an allgemeiner Melanose. IX. Forgusson ${ }^{18}$ ). Gestieltes, ulcerirtes, stark blutendes, melanotisches Sarcom der Haut des Mons veneris. Abbindung; nach 2 Jahren molanotische Inguinaldrüsen entfernt. X. H'ischer ${ }^{19}$ ): 54 jährige Frau, bei der früher ein schwarzes Gewächs am Damm abgebunden war und welche jetzt ein Melanosarcom der linken Inguinaldrüsen zeigt. Exstirpation; nach 11 Jahren kein Recidiv. XII. Blümke ${ }^{2 D}$ ) 71 jährige Frau, in die Klinik aufgenommen am 17. 2. 90. Operation durch Kallenbach; dic geschwollenen Lymphdrüsen werden nicht mit entfernt. Tod 1. 12. 90. XIIL. Haeckel21): 69 jährige Frau zeigt cinen massig-festen, faustgrossen, nit Epithel überall bekleideten Tumor von dunkelblauer Farbe mit weisslich marmorirter Zeichmung an der Oberfläche, die ganze kleine Labie, die Clitoris und den obersten Theil der rechten Nymphe einnehmend und auf die beiden Iab. maj. übergreifend. Ler Tumor lässt sich für das Auge scharf, für das Gefühl nur undeutlich gegen die Um. gebung abgrenzen. Entsprechend den Corpora cavernosa elitoridis fühlt man zwei harte Stränge. Rechte und linke Inguinaldrüsen, Simon'sche Cubitaldrüse geschwollen. Am Körper keine Naevi. Frau ohne wesentliche Beschwerden. Radicaloperation durch Braun mit Ausnahme der infiltrirten Drüsen, welche links in der Tiefe des Schenkelkanals längs der grossen Gefüsse weit in die Pauchhöhle hincinziehen. 5 Monate nach der Operation Tod in Folge von Metastasen in der BauchhöhIc, namentlich der Leber, Icterus, Ascitcs, Oedemen der unteren Extremitäten. Section nicht gemacht. XIV. Behrend 1874: Dic Geschwulst nimmt die Gegend der Clitoris ein; Metastasen in der Inguinalgegend. Amputation; im weiteren Verlaufe Metastasen, Exitus. XV. M. Kaposi ${ }^{22}$ ): Melanotischer Krebs an der linken grossen Schamlippe. Es ist in dem Werke nicht an-

16) Pigmentsarcom d. äuss. Genitalien. Centralbl. f. Gynäk. 1881. No. 20.

$\left.{ }^{17}\right)$ Annales de gynécologie. Juli 1€8's. Ref. im Centralbl. f. Gynäcol. No. 51. 1886.

18) Recurence of a melauotic tumor. Removal. Lancet. 1851. Vol. I. p. 622.

i9) S. o. S. 550 .

${ }^{20)}$ Ueber maligne Tumoren der Vulva. I.-D. Halle a. S. 1891.

2) Ueber melanotische Gesehwülste der weibl, Genital. Arch. f. Gyn. 1888. Bd. 32. S. $400-409$.

$\left.{ }^{22}\right)$ Tafelwerk: Syphilis der Haut und der angrenzenden Schleimhäute.

Archir f. pathol. snat. Bd. 154. Hft. 2. 
gegeben, in welcher Kliwik der Fall heobachtet wurde, and wie rler weitere Verlan sich gestaltete. Die Abbildung zeigt am unteren Rande der grossen linken Schamlippe eine zweithalergrosse, lupusartige, schwarz-blane Excrescenz. Linke Inguinaldrüsen afficirt.

Betroffs der Eäufigkeit melanotischer Tumoren an menschlischen Körper überhaut, speciell an den Geuitalien der Frau, botreffs ihrer Prälilectionsstellen, ihrer Genese, Actiologie, Prognose, Diagnos, ihres Verlaufes und ihrer Therapie verweise ich anf meine Dissertation $\$$. 83 - 30.

\section{XVIII. \\ Ein Beitrag zur Entstehnng der Gallensteine.}

Von Professor D. Hansemann in Berlin.

Ueber dia Zeit, welche Gallensteine zu ihrer Entstehnng" gebrauchen, ist bisher eigentlich gar nichts bekannt, $j$ a es ist diese Frage von den meisten Autoren überhaupt gar nicht diskutirt worden. So findet sich weder in Frexichs' lueberkrankheiten, noch in Chareot's Maladies du foie et des reins, noch auch in Naunyn's Klinik der Cholelithiasis irgend etwas daruber. Auch sonst habe ich nirgends eine sichcre Angave darüber auffinden können. Der Grund hierfür liegt zweifellos darin, dass Gallensteine ganz symptomlos in der Gallenblase liegen kiomen und die auftretenden Symptome daher keinen Schluss auf die Zeit der Entstehung der Gallensteine zulassen. Ferner kann man einem Gallenstein nicht ansehen, ob er alt oder jung ist; weder aus seiner Grösse, noch ans seiner chemischen und morphologischen Beschaffenheit lässt sich irgend ein Gchluss anf sein Alter ziehen. Endlich ist es bisher nicht gelungen, Galleasteino kïnstlich zil erzeugen und sich dadurch eine Anschanung uber die Zeitdauer ihrer Enwicklung zu verschaffen,

Eine solche Kenntniss würde zweifellos von Werth für das Verständniss der Cholelithiasis sein. Sie würde ferner von Interesse sein für die Frage des Zusammenhangs der Gallonsteine mit Gallenblasenkrebsen. Denn so olt anch diese Frage diskutirt ist, so fehlt doch immer noch ein Beweis furr ihre Bejahung; über eine gewisse snbjective Wahrscheinlichkeit kommt man nicht hinaus. Es findea sich zwar in fast allen Fällen von Gallenblasenkrebs Gallensteine, so dass wohl in Zusammenhang giemlich sicher besteht. Indessen trage ich Bedenken, dieseu ohne Weiteres go qu deuten, dass die Gallensteine den Krebs verursachen, und zwar aus folgenden Gründen: Gallensteine finden sich in einem grossen Procentsatz bei älteren, in Carcinom-Alter stehenden Individnen, ohne dass sich ein Trebs entwickelt; in zmei Fullen von primärem Gallenblasenkrebs, die içh beobahtete, waren 